Abdalla Saad Abdalla Al-Zawi ${ }^{1,2}$ (D) Abdul Syed ${ }^{3}$, Ali Salih ${ }^{1}$, Philip Idaewor ${ }^{1}$, Mohamed Elamass ${ }^{1}$, Agnieszka Kapturek ${ }^{4}$

${ }^{1}$ Basildon \& Thurrock University Hospital, Nethermayne, Basildon, Essex, United Kingdom

${ }^{2}$ School of Medicine, Anglia Ruskin University, Chelmsford, United Kingdom

${ }^{3}$ Southend University Hospital, Prittlewell Chase, United Kingdom

${ }^{4}$ Pratia Medical Research Centre, Skórzewo, Poland

\title{
Evaluation of the correlation between Ki-67 proliferative index and the histopathological grade of invasive neoplasms in early breast cancer aged $<70$ years of age: a review of 300 cases
}

\section{Corresponding author:}

Abdalla Saad Abdalla Al-Zawi, Basildon \& Thurrock University Hospital, Nethermayne, Basildon, Essex United Kingdom,

e-mail: abdalasaad@gmail.com
Medical Research Journal 2021;

Volume 6, Number 1, 4-7

10.5603/MRJ.a2020.0049

Copyright (c) 2021 Via Medica

ISSN 2451-2591

e-ISSN 2451-4101

\begin{abstract}
Introduction: According to the WHO 2018 reports, breast cancer is the fourth most common cause of cancer deaths worldwide. The breast cancer is recognized as the most common malignancy in the UK, it accounts for about $15 \%$ of all newly diagnosed malignancies, followed by prostate (13\%), lung (13\%), and bowel (11\%). Ki-67 proliferative index became a key element in the diagnostic process of breast cancer, in addition to its role as a predictive tool and prognostic marker during the planning of adjuvant therapy as hormonal manipulation of systemic chemotherapy. The study aims to present the correlation analysis between Ki-67 and the different histological grade in breast cancer tumours.

Material and methods: A cohort of 300 patients treated for early breast cancer was included in the study. The demographic data, histopathological subtype, hormonal and HER2 (Human epidermal growth factor receptor-2) receptor status and Ki-67 were analysed. The cut-off point was set at $20 \%$ to distinguish "high Ki-67" from "low Ki-67".

Results: About 35\% $(n=106)$ of the cases were grade II with low Ki-67, $26 \%(n=79)$ grade III with high Ki-67, 19\% $(n=56)$ grade II with high Ki-67, 11\% $(n=34)$ grade I with low Ki-67. The high Ki-67 seen in 135 patients who belong to grade II and III groups (45\%), where low Ki-67 in $42 \%$ of those cases, however, grade III alone contained more cases of high Ki-67 than any other histological grade group. Conclusions: A higher tumour histopathologic grade was correlated with higher Ki-67 values $(\mathrm{OR}=7.12$, 95\% Cl 16.75-3.03; $\mathrm{p}<0.0001)$.

Key words: Ki-67, breast cancer, mammography, chemotherapy, cell cycle
\end{abstract}

Med Res J 2021; 6 (1): 4-7

\section{Introduction}

Worldwide, one in fourteen women will develop breast cancer at the age of 0-79; this figure will rise to one in 9 women in developed countries. WHO reports revealed that breast cancer is the second most frequent malignancy worldwide where lung cancer is the most frequent one. Also, breast cancer is occupying the $5^{\text {th }}$ place in cancer-related mortality list where it comes after lung, colon, stomach and liver cancer. Ki-67 pro- tein proliferative index expression is correlated to the proliferative activity of malignant tumour cells, allowing it to be used as an indicative marker of tumour aggressiveness [1]. Ki-67 expression assessment in the early breast cancer increasingly has become an essential diagnostic tool in adjuvant treatment planning, particularly during the indication of upfront chemotherapy to hormone-sensitive tumour patients. The study aims to present the correlation analysis between Ki-67 and the different histological grade in breast cancer tumours. 


\section{Material and methods}

A cohort of 300 patients treated for early breast cancer in the period between 2016-2020 was included in the study. Age ranges were between 27-67 years. The demographic data, histopathological

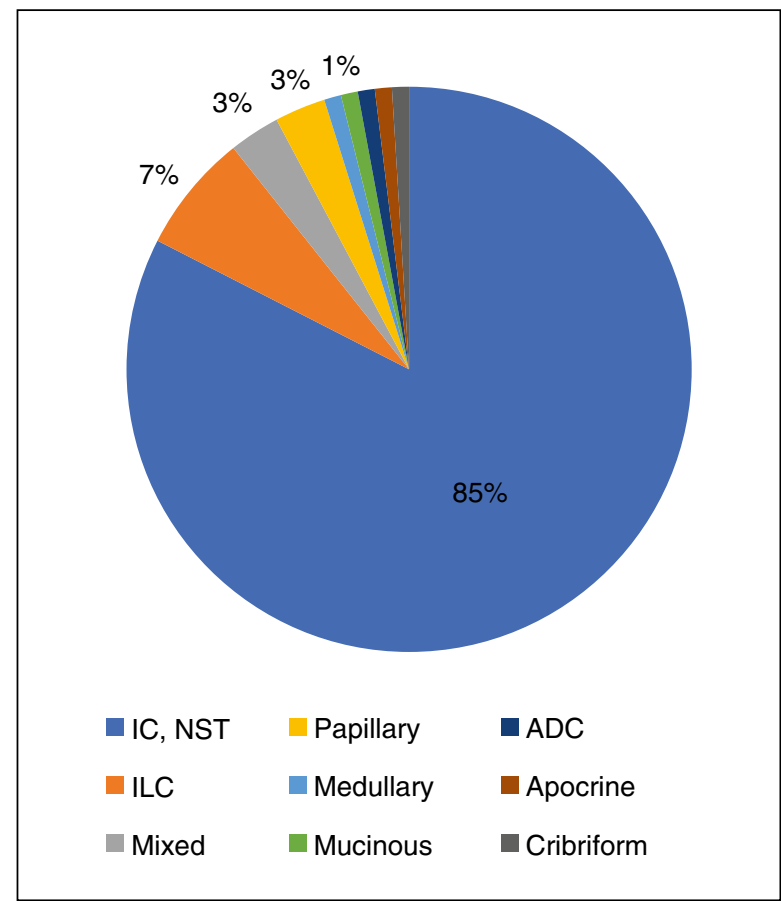

Figure 1. The histopathological subtypes of 300 cases of early breast cancer. IC, NST - invasive ductal carcinoma of no special type; ILC — invasive lobular carcinoma; ADC - adenoid cystic carcinoma subtype, hormonal and HER2 receptor status and $\mathrm{Ki}-67$ were analysed. The Ki-67 results were dichotomized by a cut-off point of $20 \%$ into "low Ki-67 $\leq 20 \%$ " and "high Ki-67 $\geq 20 \%$ ".

\section{Results}

Majority of histological subtype (85\%) was invasive carcinoma of no special type (Fig. 1). About $52 \%$ of the IC, NST (Invasive carcinoma of no special type) has Ki67 higher than $20 \%$ (Fig. 2). About 35\% ( $n=106)$ of the cases were grade II with low Ki-67, 26\% $(n=79)$ grade III with high Ki-67, $19 \%(n=56)$ grade II with high $\mathrm{Ki}-67,11 \%(\mathrm{n}=34)$ grade I with low Ki-67 (Tab. 1). The high Ki-67 seen in 135 patients who belong to grade II and III groups (45\%). The same grade groups (II and III) show low Ki-67 in $42 \%$ of the cases, however, grade III alone contains more cases of high Ki-67 than any other histological grade group. Also, after merging Grade I and Grade II tumours in one group, Grade III tumours showed a significant correlation with high Ki-67 (OR = 11.96, 95\% Cl 8.28-6.94; $p<0.0001)$. Grade III tumours with $\mathrm{Ki}-67$ below $20 \%$ were less frequent and seen in only $7 \%$ of the cohort, and about $80 \%$ of all grade III tumours had a Ki-67 higher than $20 \%$. Most of the grade I tumours was associated with $\mathrm{Ki}-67$ less than $20 \%$, they accounted for about $90 \%$ of this group (Fig. 3).

\section{Discussion}

In the UK, around 150 new breast cancer cases are detected every day, and this results in around

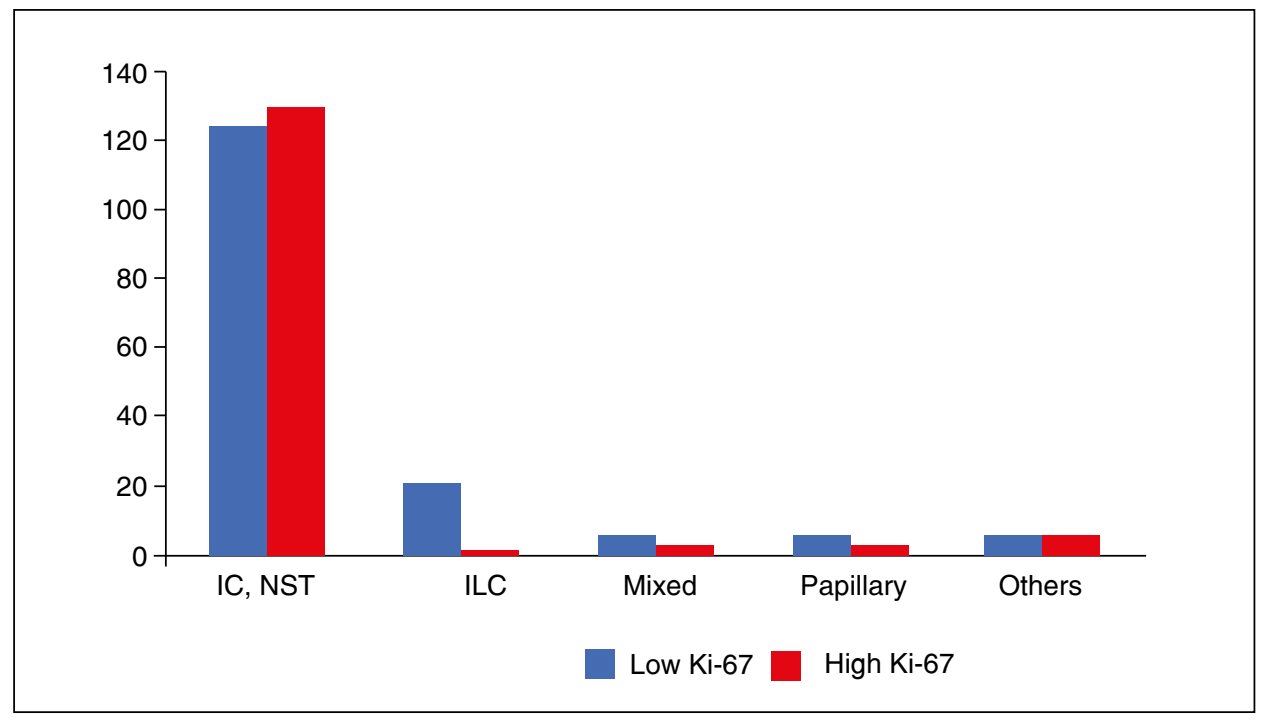

Figure 2. Ki-67 proliferative index among different histopathological subtypes. IC, NST — invasive ductal carcinoma of no special type; ILC — invasive lobular carcinoma; ADC — adenoid cystic carcinoma; Low Ki-67: < 20\%; High Ki-67: $\geq 20 \%$ 
Table 1. Ki- $67 \%$ expression and tumour grade in 300 cases of breast cancer

\begin{tabular}{lccc}
\hline Ki-67 level & Grade I & Grade II & Grade III \\
\hline Low Ki-67 $\leq 20 \%$ & $11 \%(34)$ & $35 \%(106)$ & $07 \%(21)$ \\
High Ki-67 $>20 \%$ & $01 \%(4)$ & $18 \%(56)$ & $26 \%(79)$ \\
\hline
\end{tabular}

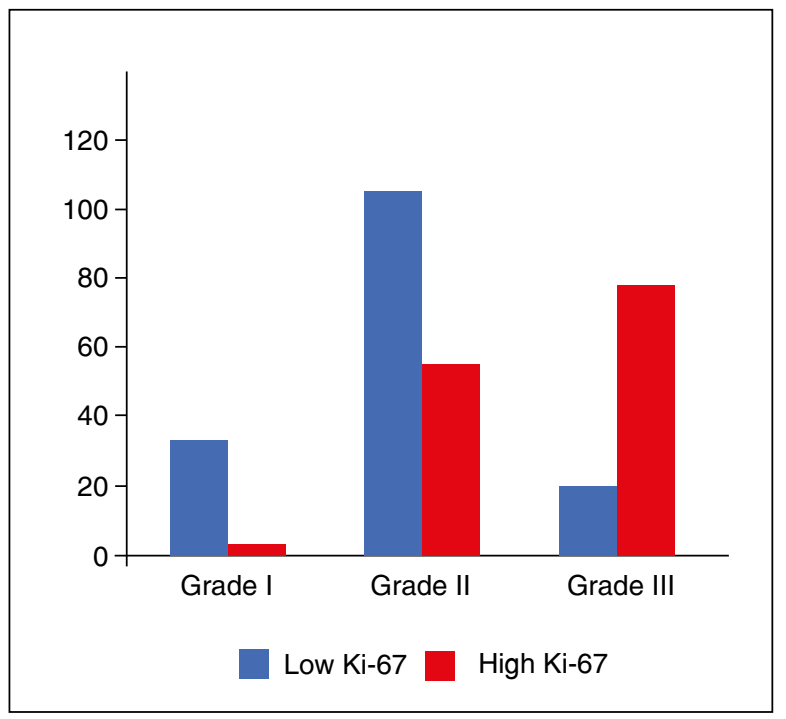

Figure 3. Tumour grade and Ki-67 proliferation index in 300 cases of early breast cancer, higher tumour grades associated with high Ki-67 values. Low Ki-67: < 20\%; High Ki-67: $>20 \%$

55,200 new invasive breast cancer cases diagnosed every year and makes it the most common female cancer in the UK. In the United States, breast cancer is also reported to be the most common female cancer, where it accounts for $30 \%$ of all newly diagnosed cancer among females [2].

As breast cancer is regarded as a common malignancy worldwide [1], many countries adopted the 3-yearly breast cancer screening mammogram policy (Fig. 4). Every woman which belongs to the age group between $50-70$ years is invited for a screening mammogram every 3 years. Every woman above 70 years can still have the screening mammogram every 3 years but won't automatically be invited. This has to be arranged on request through the family doctor or the local breast screening unit. The screening results in increased rates of early diagnosis and allows the clinician to commence the treatment early. This will have a positive impact on the management outcome.

The clinical course of breast invasive carcinoma is very heterogeneous because the variable biomarker factors impact its behaviour. Assessment of clinical and molecular markers is helpful during the decision making when choosing the most appropriate treatment

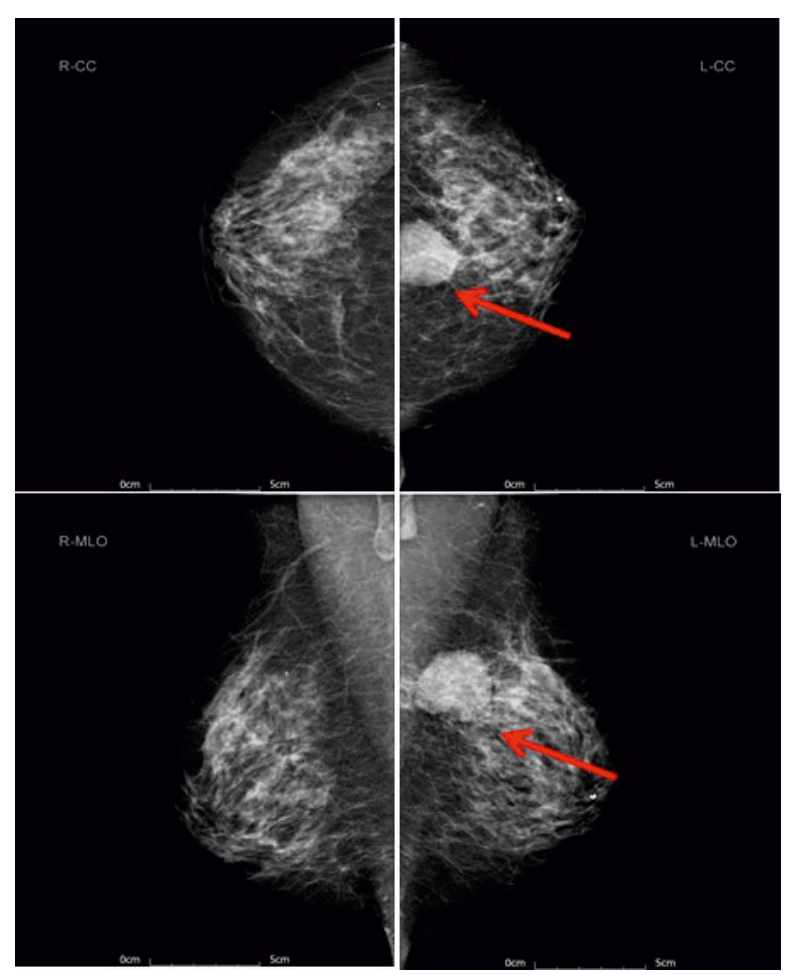

Figure 4. MLO (Mediolateral Oblique) and CC (Craniocaudal) views of the mammogram show a soft tissue density with fairly well-defined margins seen in the left upper inner quadrant close to the midline; the appearance is suspicious and measures about $3.7 \mathrm{~cm}$ ( red arrow); there are also bilateral benign calcific foci

modality. The Ki-67 nuclear antigen was identified in the 1980s, as a protein which is related to cell cycle, and only detected in active dividing cell phases (G1-, S-, G2- and M-phase) but not in quiescent cells (G0 phase) [3]. Ki-67 expression assessment in the early breast cancer increasingly has become an essential diagnostic tool in adjuvant treatment planning, particularly during the indication of upfront chemotherapy to hormone-sensitive tumour patients as it is considered as a dynamic biomarker of treatment efficacy of neoadjuvant therapy [4]. According to the oestrogen receptor (ER), progesterone receptor (PR), and $\mathrm{Hu}$ man Epidermal Growth Factor Receptor 2 (HER2), the main breast cancer subtypes have been identified. The first subtype is luminal type $\mathrm{A}$, which is $\mathrm{ER}^{+}$and/or $\mathrm{PR}^{+}$/ /HER2 ${ }^{-}$status, low-grade tumour, and characterised by a good prognosis [5]. The second subtype is lumi- 
nal $\mathrm{B}$, it is $\mathrm{ER}^{+}$and/or PR/HER2- status and accounts for about $10 \%$ of all breast invasive cancers [6]. The third subtype is luminal B-like (or HER2 positive disease), its immunohistochemistry profile is $\mathrm{ER}^{+}$, HER2 overexpression or amplification, and any Ki-67or PR [7]. The fourth subtype is HER2 enriched tumour (HER2-2E), they are HER2+, with high expression of ERBB2 and genes of the 17q amplicon, such as GRB7, and low to an intermediate expression of luminal genes such as ESR1 and PGR. Majority of HER2-E tumours are hormone receptor-negative (HR-), however, $\sim 30 \%$ are typically HR+ [8]. Patient age, tumour histological subtype, tumour size, female sex hormone receptor, human epidermal growth factor receptor 2 (Her2neu), presence of lymph node disease, presence of genetic mutation as well as Ki-67 has both predictive and prognostic impact on breast cancer management, especially in the loco-regional disease $[9,10]$. The histological grade itself is an essential determining factor of breast cancer prognostic tools and is integrated into the staging assessment as in NPI algorithms (Nottingham Prognostic Index) to allow choosing the most optimal therapy for patients with breast cancer. The tumour histological grade is evaluated by examining the morphological features as mitotic count, tubule formation and nuclear pleomorphism. This may highlight the hypothesis of the histological grade indirectly related to Ki-67 based on the mitotic count [2]. This study is limited by the small cohort number, however, the data obtained allow us to suggest the importance of routine use of Ki-67 measurement in the diagnostic workup of breast cancer, to determine breast cancer histological subtypes and also to use it as a prognostic and a predictive tool in the disease management $[11,12]$. In the presented study, the correlation between $\mathrm{Ki}-67$ level and the tumour histopathologic grade variables was analysed. Higher tumour histopathologic grade was correlated with higher Ki-67 values. Tumours of grade III with a Ki-67 below $20 \%$ were less frequent. On the other hand, most grade I tumours tend to possess Ki-67 below $20 \%$.

\section{Conclusions}

In this study, it was observed that Ki-67 is to be considered a valuable biomarker in breast cancer patients. A higher tumour histopathologic grade was correlated with higher Ki-67 levels.

\section{Key points}

The Ki-67 level is considered a valuable biomarker in breast cancer management especially in its role as a prognostic indicator. Ki-67 expression is associated with common histopathological parameters as the tumour histological grade, higher ki67 correlates with higher tumour grade $(\mathrm{OR}=7.12,95 \% \mathrm{Cl}$ 16.75-3.03; $\mathrm{p}<0.0001)$. Measuring Ki-67 index expression would have a significant impact on adjuvant or neoadjuvant chemotherapy decision.

\section{References}

1. Saad Abdalla Al-Zawi A, Syed A. Ki67 Proliferation index as a prognostic and predictive tool for pathological response after upfront chemotherapy in breast cancer. Paripex Indian Journal of Research. 2020; $9 ; 11$

2. Liang Q, Ma D, Gao RF, et al. Effect of Ki-67 Expression Levels and Histological Grade on Breast Cancer Early Relapse in Patients with Different Immunohistochemical-based Subtypes. Sci Rep. 2020; 10(1): 7648, doi: 10.1038/s41598-020-64523-1, indexed in Pubmed: 32376868

3. Miller I, Min M, Yang C, et al. Ki67 is a Graded Rather than a Binary Marker of Proliferation versus Quiescence. Cell Rep. 2018; 24(5): 1105-1112. e5, doi: 10.1016/j.celrep.2018.06.110, indexed in Pubmed: 30067968.

4. Maranta AF, Broder S, Fritzsche C, et al. Do you know the Ki-67 index of your breast cancer patients? Knowledge of your institution's Ki-67 index distribution and its robustness is essential for decision-making in early breast cancer. Breast. 2020; 51: 120-126, doi: 10.1016/i. breast.2020.03.005, indexed in Pubmed: 32302928.

5. Soliman NA, Yussif SM. Ki-67 as a prognostic marker according to breast cancer molecular subtype. Cancer Biol Med. 2016; 13(4): 496-504, doi: 10.20892/j.issn.2095-3941.2016.0066, indexed in Pubmed: 28154782.

6. Bustreo S, Osella-Abate S, Cassoni P, et al. Optimal Ki67 cut-off for luminal breast cancer prognostic evaluation: a large case series study with a long-term follow-up. Breast Cancer Res Treat. 2016; 157(2): 363371, doi: 10.1007/s10549-016-3817-9, indexed in Pubmed: 27155668.

7. Inic Z, Zegarac M, Inic M, et al. Difference between Luminal A and Luminal B Subtypes According to Ki-67, Tumor Size, and Progesterone Receptor Negativity Providing Prognostic Information. Clin Med Insights Oncol. 2014; 8: 107-111, doi: 10.4137/CMO.S18006, indexed in Pubmed: 25249766

8. Prat A, Pascual T, Adamo B. Intrinsic molecular subtypes of HER2 + breast cancer. Oncotarget. 2017; 8(43): 73362-73363, doi: 10.18632/oncotarget.20629, indexed in Pubmed: 29088709.

9. Hashmi AA, Hashmi KA, Irfan M, et al. Ki67 index in intrinsic breast cancer subtypes and its association with prognostic parameters. BMC Res Notes. 2019; 12(1): 605, doi: 10.1186/s13104-019-4653-x, indexed in Pubmed: 31547858.

10. Kanyılmaz G, Yavuz BB, Aktan M, et al. Prognostic Importance of Ki-67 in Breast Cancer and Its Relationship with Other Prognostic Factors. Eur J Breast Health. 2019; 15(4): 256-261, doi: 10.5152/ejbh.2019.4778, indexed in Pubmed: 31620685

11. Petric M, Martinez S, Acevedo F, et al. Correlation between Ki67 and histological grade in breast cancer patients treated with preoperative chemotherapy. Asian Pac J Cancer Prev. 2014; 15(23): 10277-10280, doi: 10.7314/apicp.2014.15.23.10277, indexed in Pubmed: 25556461.

12. Al-Zawi A. Ki -67 proliferative index as a predictive tool for axillary pathological complete response in node-positive breast cancer. International Journal of Medical Science. 2020; 7(11): 1-4, doi: 10.14445/23939117/ijms-v7i11p101 